\title{
Treatment of vascular activity secondary to atypical choroidal nevus using intravitreal bevacizumab
}

\author{
This article was published in the following Dove Press journal: \\ Clinical Ophthalmology \\ 22 July 2014 \\ Number of times this article has been viewed
}

\author{
Milena L Cavalcante' \\ Victor MVillegas ${ }^{2}$ \\ Aaron S Gold ${ }^{2}$ \\ Ludimila L Cavalcante' \\ Marcela Lonngi' \\ Nisha V Shah' \\ Timothy G Murray ${ }^{2}$ \\ 'Bascom Palmer Eye Institute, \\ University of Miami Miller School \\ of Medicine, Miami, FL, USA; \\ ${ }^{2}$ Murray Ocular Oncology and Retina, \\ Miami, FL, USA
}

Objective: To report the optical coherence tomography (OCT) findings of 27 eyes treated with intravitreal bevacizumab for intraretinal and subretinal vascular activity associated with atypical choroidal nevi.

Methods: This was an Internal Review Board-approved retrospective review of 27 eyes of 27 patients with choroidal nevus treated for secondary vascular activity with intravitreal injections of bevacizumab, performed by a single surgeon (TGM) at the Bascom Palmer Eye Institute. All patients were rigorously evaluated before the procedure and followed thereafter with ophthalmic examinations, refractive analysis, fundus photos, optical coherence tomography (OCT), and ocular echography. Patient demographics, tumor characteristics, dates of bevacizumab injections, and spectral-domain (SD)-OCT findings at each injection were recorded. Macular edema was graded as per SD-OCT findings for the initial and final visit.

Results: The mean age was 66.6 years (range, $40-86$ years), with ten males and 17 females. Mean, median, and range baseline best corrected visual acuity (BCVA) were 20/53, 20/40, and 20/20-4/200, respectively. After a mean follow up of 29 months, the final BCVA mean, median, and range were 20/50, 20/40, and 20/20-20/400, respectively. The final BCVA ranged from $20 / 20$ to 20/25 in nine eyes, while only six eyes had an initial BCVA within the same range. All patients demonstrated OCT findings of vascular activity suggestive of choroidal neovascularization (CNV). Initial SD-OCT findings included intraretinal cysts in eleven eyes, intraretinal fluid in six eyes, subretinal fluid in 14 eyes, pigment epithelial detachment in six eyes, epiretinal membrane in five eyes, and subretinal neovascularization in 14 eyes. On fundus photos, four eyes presented retinal hemorrhage. A mean of eight (range of 1-31) intravitreal bevacizumab $(1.25 \mathrm{mg} / 0.05 \mathrm{cc})$ injections were given in all cases. A total of $37 \%(10 / 27)$ of eyes had complete or partial regression of vascular activity. The mean initial OCT classification for macular edema was 3 and a mean grade of 3 was maintained at the final follow-up OCT. All 27 choroidal nevi remained stable, and there were no adverse effects from the bevacizumab injections.

Conclusion: To our knowledge, this is the largest published case series of eyes treated with intravitreal bevacizumab for vascular activity associated with choroidal nevus. Intravitreal bevacizumab seems to be effective in the treatment of CNV secondary to choroidal nevus, and OCT can be a useful tool in the follow up of these patients, to assess the regression of CNV and to monitor macular edema.

Keywords: macular edema, choroidal neovascularization, subretinal fluid, optical coherence tomography

\section{Introduction}

Choroidal nevi are benign, pigmented or nonpigmented intraocular tumors that are an incidental finding of routine examinations. Studies have suggested that these lesions are particularly frequent in Caucasians, presenting in $6.5 \%$ of the general white population, and are suspected precursors of choroidal melanoma. ${ }^{1,2}$ Although growth is not an absolute indicator of malignancy, many ophthalmologists observe these lesions for size
Correspondence:Timothy G Murray Murray Ocular Oncology and Retina, 6705 Red Road, Suite 4I2, Miami, FL 33143, USA

Tel +I 3054877470

Fax + I 786326 6I47

Email tmurray@murraymd.com 
alteration since according to histopathologic review, small pigmented lesions that grow are more likely to be malignant melanoma than benign nevi. ${ }^{3}$

The alterations induced by choroidal nevi include secondary changes of the pigment epithelium that can lead to the formation of drusen, or even vascular activity in the form of intraretinal fluid (IRF) or subretinal fluid (SRF) and proliferation of choroidal neovascularization (CNV). ${ }^{4,5}$ Studies have shown that the presence of vascular activity over a nevus is uncommon and that this is not a sole indicator of malignant transformation but that it can be responsible for loss of visual acuity (VA) due to leakage, in which case treatment is indicated. ${ }^{5,6}$

Amongst the treatment modalities for vascular activity associated with atypical choroidal nevi, laser photocoagulation has been reported to be effective when neovascularization is extrafoveal. Nevertheless when vascular activity is subfoveal, alternative treatment, such as photodynamic therapy with verteporfin (PDT), is considered in order to spare the foveal center..$^{7-9}$ One case report also described transpupillary thermotherapy as a viable option for subfoveal CNV associated with a nevus. ${ }^{10}$ In addition, the anti-vascular endothelial growth factor (VEGF) agent bevacizumab has shown favorable outcomes in the treatment of subfoveal CNV secondary to multifocal choroiditis, angioid streaks, and myopic degeneration ${ }^{11}$ and has been successfully utilized in the management of CNV associated with atypical choroidal nevi. ${ }^{12}$

Lately, spectral-domain optical coherence tomography (SD-OCT) has been used to evaluate retinal alterations and grade the level of macular edema. SD-OCT provides a two-dimensional, cross-sectional, high-resolution image of ocular tissues and demonstrates higher sensitivity than clinical examination to detect retinal pigment epithelium alterations from a nevus. ${ }^{13,14}$ In this study, we present our experience with SD-OCT in the evaluation of 27 eyes with vascular activity secondary to an atypical choroidal nevus, treated with intravitreal bevacizumab. To our knowledge, this is the largest published series.

\section{Methods}

The study was approved by the Institutional Review Board of the University of Miami and was compliant with the Health Insurance Portability and Accountability Act. ${ }^{15}$ The inclusion criteria incorporated patients with vascular activity associated with atypical choroidal nevi, injected with the vascular targeting agent bevacizumab, between September 2008 and May 2012, by the same surgeon (TGM) in the outpatient clinic at the Bascom Palmer Eye Institute, University of Miami Miller School of Medicine.
Standard injection, treatment, and follow-up protocols were followed for all intravitreal injections in this study. Informed consent was obtained and off-label use discussed in detail with each patient before injection. Preparation of the eyes before the injection was performed by a trained registered nurse following the standard preinjection protocol for the Bascom Palmer Eye Institute. This consisted of instilling 5\% povidone-iodine and $4 \%$ lidocaine drops from single-use dispensers into each conjunctiva and then, cleaning the eyelid skin and lashes with $10 \%$ povidone-iodine swabs. A sterile wire lid speculum was then inserted and lashes directed away from the eye. Three alternating cycles of povidone-iodine and lidocaine drops were instilled to the inferior fornix and conjunctiva, and a lidocaine-soaked cotton tip applicator was applied using pressure to the injection site. Injection was performed with the physician wearing sterile gloves. Each patient received $1.25 \mathrm{mg} / 0.05 \mathrm{~mL}$ of bevacizumab intravitreally through the pars plana in each eye, followed by application of one drop of gentamicin (an aminoglycoside). Patients were instructed to use postinjection antibiotics (gentamicin) four times a day for 4 days; they were not draped for the injection and did not receive antibiotics prior to injection.

All patients were rigorously evaluated before the procedure and followed thereafter with clinical eye examinations, refractive analysis, fundus photos, optical coherence tomography (OCT) (Spectralis ${ }^{\circledR}$, Heidelburg Engineering, Heidelburg, Germany), and ocular echography (Eye Cubed ${ }^{\mathrm{TM}}$, Ellex Medical Pty. Ltd., Adelaide, SA, Australia). A standard follow-up protocol was used postinjection and consisted of reevaluation of the patient 4-6 weeks later during a clinical visit, where all the aforementioned exams were performed for each patient.

Regression of vascular activity was evaluated by OCT and determined to be complete, by the resolution of all SRF and macular edema; or partial, by the presence of residual SRF or macular edema (Figures 1 and 2). OCT classification was composed of grades $1-6$, where grade 1 represented extrafoveolar, noncystoid edema; grade 2, extrafoveolar cystoid edema; grade 3, foveolar noncystoid edema; grade 4, mild-to-moderate foveolar cystoid edema; grade 5 , severe foveolar cystoid edema; and grade 6, severe foveal cystoid edema with SRF. ${ }^{16}$

\section{Results}

Eyes with vascular activity associated with a choroidal nevus $(n=27)$ were treated with intravitreal bevacizumab. The mean patient age was 66.6 years (range, $40-86$ years), with ten males and 17 females included in the sample. A total of 12 nevi were in the right eye, while 15 were in the left eye. Mean initial nevus height by ultrasonography (mm) was 1.36 . The nevus location included macular involvement in 51.9\% 


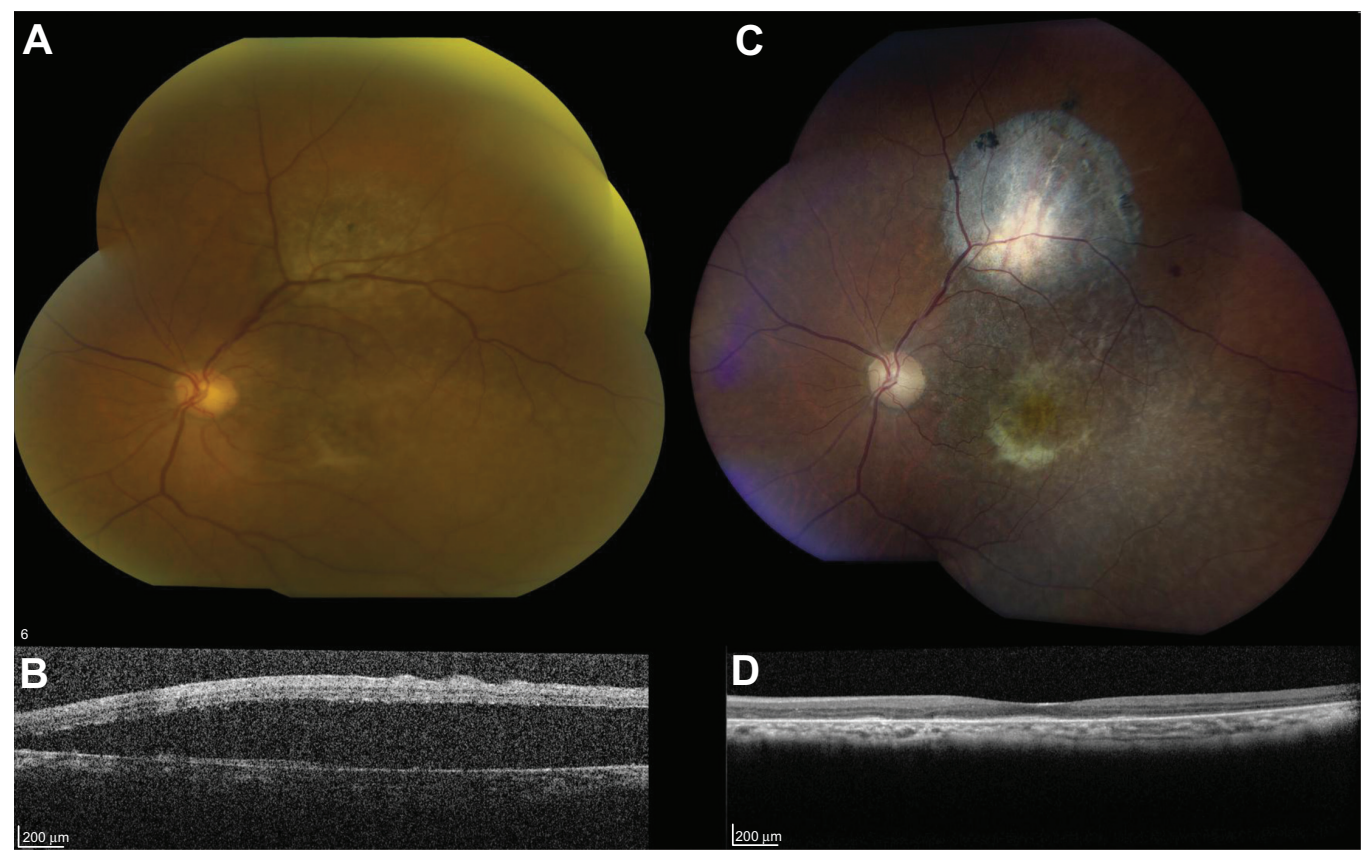

Figure I 83-year-old male with 20/200 vision in the left eye.

Notes: (A) Initial fundus photograph shows an atypical pigmented choroidal nevus, with presence of drusen. (B) Initial optical coherence tomography demonstrates considerable subretinal fluid. (C and D) Imaging after seven bevacizumab injections, with complete regression of fluid. Best corrected final visual acuity was maintained at $20 / 200$ at 16 months of follow-up.

(14/27) of eyes. All 27 choroidal nevi remained stable after receiving a mean of eight intravitreal bevacizumab injections (range of 1-31). Patients were followed for a mean/median/ range of 29/22/2-88 months. Additional patient features are listed in Table 1.

All patients $(n=27)$ had OCT findings associated with vascular activity from presumed CNV. Initial OCT findings included intraretinal cysts in eleven eyes (40.7\%), IRF in six eyes (22.2\%), SRF in 14 eyes (51.9\%), pigment epithelial detachment (PED) in six eyes $(22.2 \%)$, epiretinal membrane in five eyes $(18.5 \%)$, and subretinal neovascularization in 14 eyes $(51.9 \%)$. Final OCT findings included intraretinal cysts in 15 eyes $(55.6 \%)$, IRF in five eyes (18.5\%), SRF in 16 eyes $(59.3 \%)$, PED in three eyes $(11.1 \%)$, epiretinal

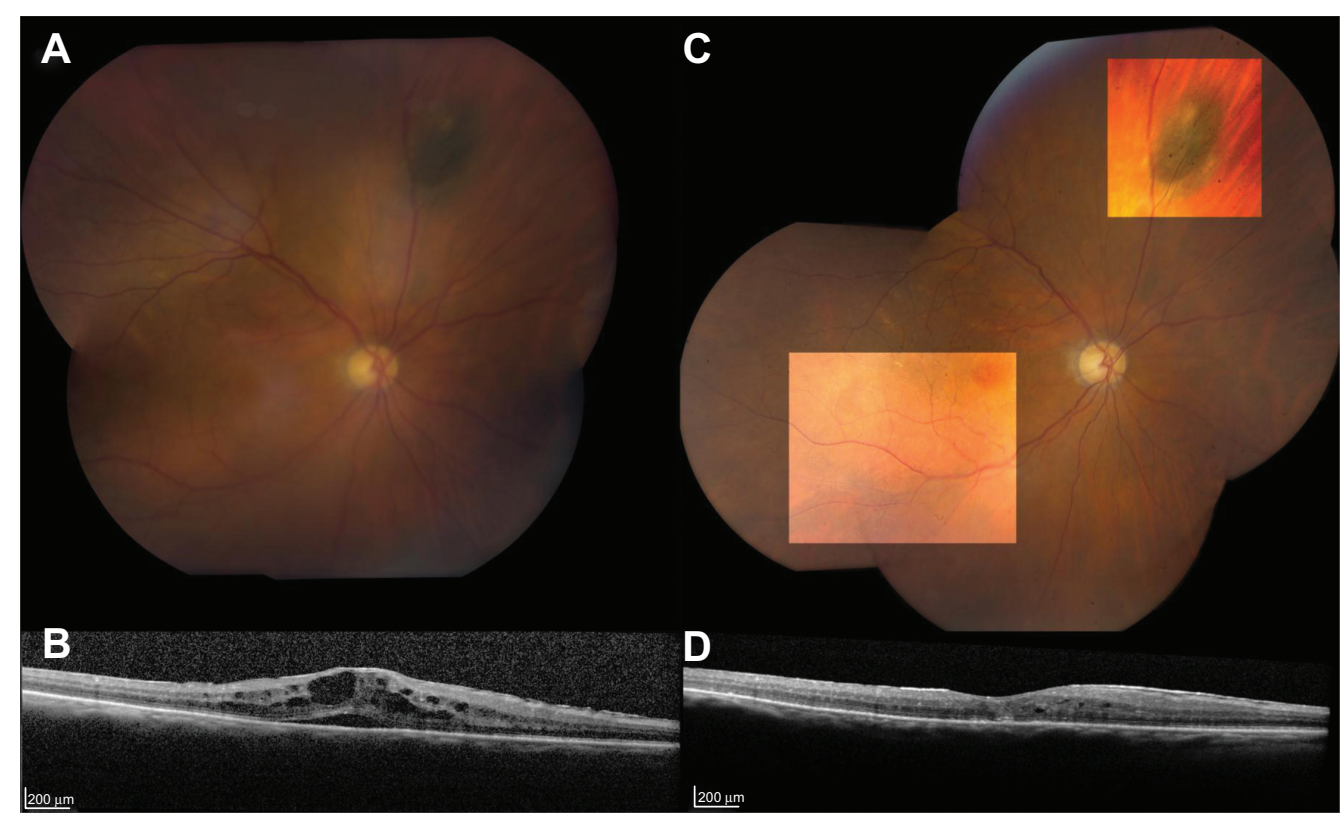

Figure 2 83-year-old female with 20/200 vision in the right eye.

Notes: (A) Initial fundus photograph shows a pigmented choroidal nevus, with presence of drusen. (B) Initial optical coherence tomography demonstrates subretinal fluid and intraretinal cysts. (C and D) Imaging after 18 bevacizumab injections, showing partial regression of the fluid. Best corrected visual acuity was maintained at $20 / 200$ at 47 months of follow up. 


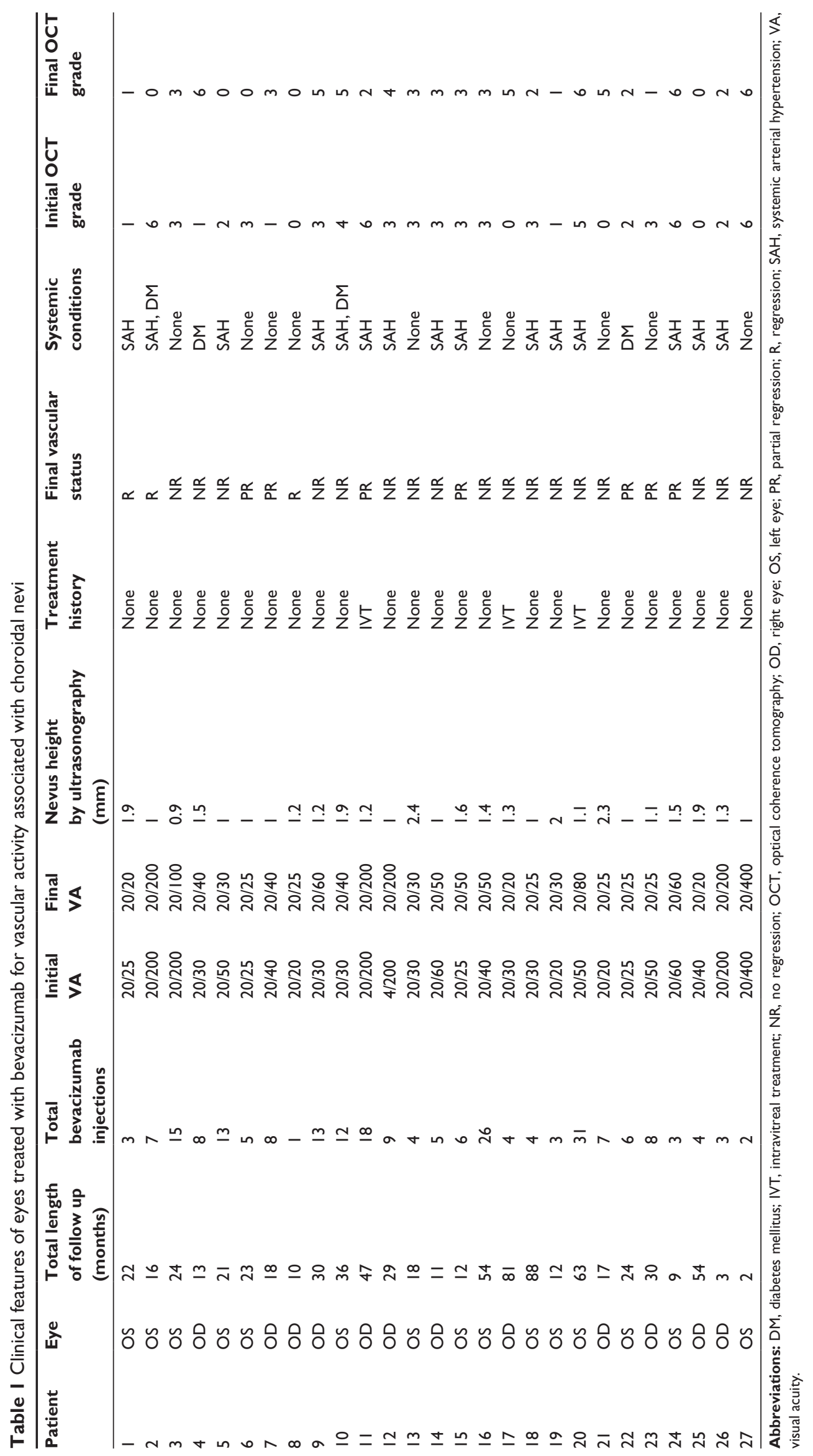


membrane in six eyes (22.2\%), and subretinal neovascularization in 15 eyes $(55.6 \%)$. In addition, final OCT findings in patients with worse final best corrected visual acuity (BCVA) (9/27) included intraretinal cysts in $55.6 \%$ of eyes, IRF in $22.2 \%$ of eyes, SRF in $77.8 \%$ of eyes, PED in $11.1 \%$ of eyes, epiretinal membrane in $22.2 \%$ of eyes, and subretinal neovascularization in $44.4 \%$ of eyes. Final central macular thickness was decreased or maintained in $30 \%$ of eyes $(8 / 27)$. Initial fundus photos showed retinal hemorrhage in four eyes $(14.8 \%)$, while final ones showed retinal hemorrhage in two eyes (7.4\%).

Mean, median, and range baseline BCVA were 20/53, 20/40, and 20/20-4/200, respectively; the initial BCVA ranged from 20/20-20/50 in 19 eyes, 20/60-20/100 in two eyes, and 20/200 or worse in six eyes. At last follow up, mean, median, and range BCVA were 20/50, 20/40, and 20/20-20/400, respectively; the final BCVA ranged from 20/20-20/50 in 18 eyes, 20/60-20/100 in four eyes, and $20 / 200$ or worse in five eyes. However, nine eyes had a final BCVA ranging from 20/20-20/25, while only six eyes had an initial BCVA within the same range.

Mean initial macular edema grade was also maintained at grade 3 after a mean of 29 months of treatment. Specifically, 70.4\% (19/27) of eyes maintained or improved the grade of macular edema, while $66.7 \%$ (18/27) of eyes maintained or improved BCVA at the final visit. A total $37 \%$ of eyes (10/27) showed improvement of vascular activity-associated OCT findings after bevacizumab injection. On final visit, three eyes demonstrated complete resolution of SRF, and seven eyes showed partial regression.

\section{Discussion}

The main concerns with an atypical choroidal nevi are the possibility of malignant transformation and the risk of inducing VA loss. A case series of 3,422 eyes with choroidal nevi, by Shields et al reported a vision loss at 15 years in $2 \%$ of eyes with an extrafoveolar nevus and in $26 \%$ of eyes with a subfoveolar nevus. This study showed that the most important factor leading to poor final VA was foveal edema, whereas the most important risk factor for VA loss was overlying PED. ${ }^{17}$ In our study, patients who experienced decline in their BCVA after 2 years initially had a macular edema of grade 2 that worsened to grade 4 , which corresponds to mildto-moderate foveolar cystoid edema. This further suggests that foveal edema contributes to poor final BCVA in patients with vascular activity associated with atypical nevi.

In a retrospective study that analyzed the OCT findings of 120 patients with choroidal nevi, PED overlying the nevus was found in $12 \%$ of cases but could be visualized clinically in only $2 \%,{ }^{13}$ suggesting the sensitivity of OCT in predicting visual outcomes. Another SD-OCT imaging study of nevi showed that the OCT enabled a more precise and reduced measurement of tumor thickness compared with ultrasonography. ${ }^{18}$ In addition, SRF is an important factor that is predictive of a melanocytic choroidal lesion and can be detected by OCT when overlooked clinically and ultrasonographically. ${ }^{19,20}$ Furthermore, we have shown that amongst the vascular activity-associated OCT findings in patients with atypical nevi, the presence of IRF and/or SRF may be more likely to lead to deterioration in VA.

Factors predictive of choroidal nevus transformation into melanoma include thickness greater than $2 \mathrm{~mm}$, the presence of SRF, orange pigment, juxtapapillary location, and symptoms of blurred vision or photopsia. ${ }^{2,21}$ All 27 nevi in this study remained stable.

Treatment of CNV associated with a choroidal nevus has been successful with laser photocoagulation therapy, when extrafoveal. ${ }^{4}$ However, the presence of a subfoveal $\mathrm{CNV}$ is a contraindication to laser photocoagulation, due to the possibility of vision-compromising scarring. Hence, alternative treatment modalities, such as $\mathrm{PDT}^{7-9}$ or transpupillary thermotherapy have been used. ${ }^{10}$ However, a study by García-Arumí et al showed that $18 \%$ of eyes with nevi treated with PDT for symptomatic SRF extending to the fovea showed an increase in tumor thickness. ${ }^{22}$ The authors suggested that PDT might not allow a good local tumor control in these cases, although there was partial resolution of SRF and improvement in VA. Nonetheless, PDT has been shown to be effective in the management of serous retinal detachment associated with a nevus, ${ }^{23}$ as well as in the treatment of extrafoveal CNV associated with a nevus. ${ }^{24}$

Bevacizumab is a recombinant humanized anti-VEGF monoclonal antibody, approved by the Food and Drug Administration as an antiangiogenic agent for the treatment of metastatic colon cancer in combination with chemotherapy. Among the most common indications for intravitreal injection of bevacizumab are radiation retinopathy, neovascular age-related macular degeneration, and presence of choroidal neovascular membranes. ${ }^{25}$ Bevacizumab has been successfully used in the treatment of CNV associated with nevi, as demonstrated by Chiang et al in a recent case series of ten patients. ${ }^{12}$ In the same series, nine patients presented with subfoveolar fluid, five with exudation, and four with hemorrhage. There was regression of CNV in all ten patients, using two to 14 injections, and $60 \%$ (6/10) of patients had improved final VA. In our study, bevacizumab treatment resulted in improvement of vascular activity in $37 \%(10 / 27)$ of eyes at the most recent follow-up as well as improved or maintained final VA in $66.7 \%(18 / 27)$ of eyes. In the present study, there was 
also variation in the number of injections needed to achieve vascular activity regression, from one to 31 injections. This is in accordance with the previous study ${ }^{12}$ and suggests that CNV associated with a nevus can have a variable degree of activity. In addition, PDT and/or conventional laser were not administered to any patient in this study.

The limitations of this study include its retrospective nature and lack of control group. Treatment guidelines are likely to continue being influenced by small studies, due to the low prevalence of vascular activity associated with nevi. In conclusion, this study suggests that intravitreal bevacizumab is effective in the treatment of vascular activity from presumed CNV associated with atypical choroidal nevi and that SD-OCT can be a useful tool in the follow-up of these patients, in order to assess the regression of vascular activity and monitoring of macular edema. We believe that the classification of macular edema can serve as a tool to predict final BCVA following bevacizumab therapy. Further studies with long-term follow-up are needed to confirm bevacizumab monotherapy as an effective treatment for patients with retinal and subretinal vascular activity associated with a nevus. To our knowledge, this is the largest published series that investigates the role of intravitreal bevacizumab in vascular activity associated with an atypical choroidal nevus.

\section{Disclosure}

The authors report no conflicts of interest in this work.

\section{References}

1. Mashayekhi A, Siu S, Shields CL, Shields JA. Slow enlargement of choroidal nevi: a long-term follow-up study. Ophthalmology. 2011; 118(2):382-388.

2. Say EA, Shah SU, Ferenczy S, Shields CL. Optical coherence tomography of retinal and choroidal tumors. J Ophthalmol. 2012; 2012:385058.

3. Factors predictive of growth and treatment of small choroidal melanoma: COMS Report No. 5. The Collaborative Ocular Melanoma Study Group. Arch Ophthalmol. 1997;115(12):1537-1544.

4. Zografos L, Mantel I, Schalenbourg A. Subretinal choroidal neovascularization associated with choroidal nevus. Eur J Ophthalmol. 2004;14(2):123-131.

5. Slusher M, Weaver RG. Presumed choroidal naevi and sensory retinal detachment. Br J Ophthalmol. 1977;61(6):414-416.

6. Callanan DG, Lewis ML, Byrne SF, Gass JD. Choroidal neovascularization associated with choroidal nevi. Arch Ophthalmol. 1993;111(6): 789-794.

Clinical Ophthalmology

\section{Publish your work in this journal}

Clinical Ophthalmology is an international, peer-reviewed journal covering all subspecialties within ophthalmology. Key topics include: Optometry; Visual science; Pharmacology and drug therapy in eye diseases; Basic Sciences; Primary and Secondary eye care; Patient Safety and Quality of Care Improvements. This journal is indexed on Submit your manuscript here: http://www.dovepress.com/clinical-ophthalmology-journal
7. Parodi MB, Boscia F, Piermarocchi S, Ferrari TM, Furino C, Sborgia C. Variable outcome of photodynamic therapy for choroidal neovascularization associated with choroidal nevus. Retina. 2005;25(4):438-442.

8. Levy J, Shneck M, Klemperer I, Lifshitz T. Treatment of subfoveal choroidal neovascularization secondary to choroidal nevus using photodynamic therapy. Ophthalmic Surg Lasers Imaging. 2005; 36(4):343-345.

9. Stanescu D, Wattenberg S, Cohen SY. Photodynamic therapy for choroidal neovascularization secondary to choroidal nevus. Am J Ophthalmol. 2003;136(3):575-576.

10. Parodi MB. Transpupillary thermotherapy for subfoveal choroidal neovascularization associated with choroidal nevus. Am J Ophthalmol. 2004;138(6):1074-1075.

11. Chang LK, Spaide RF, Brue C, Freund KB, Klancnik JM, Slakter JS. Bevacizumab treatment for subfoveal choroidal neovascularization from causes other than age-related macular degeneration. Arch Ophthalmol. 2008;126(7):941-945.

12. Chiang A, Bianciotto C, Maguire JI, et al. Intravitreal bevacizumab for choroidal neovascularization associated with choroidal nevus. Retina. 2012;32(1):60-67.

13. Shields CL, Mashayekhi A, Materin MA, et al. Optical coherence tomography of choroidal nevus in 120 patients. Retina. 2005;25(3):243-252.

14. Muscat S, Parks S, Kemp E, Keating D. Secondary retinal changes associated with choroidal naevi and melanomas documented by optical coherence tomography. Br J Ophthalmol. 2004;88(1):120-124.

15. United States Health Insurance Portability and Accountability Act of 1996. Public Law 104-191. US States 1996;100:1936-2103.

16. Horgan N, Shields CL, Mashayekhi A, Shields JA. Classification and treatment of radiation maculopathy. Curr Opin Ophthalmol. 2010;21(3): 233-238.

17. Shields CL, Furuta M, Mashayekhi A, et al. Visual acuity in 3422 consecutive eyes with choroidal nevus. Arch Ophthalmol. 2007;125(11): 1501-1507.

18. Shah SU, Kaliki S, Shields CL, Ferenczy SR, Harmon SA, Shields JA. Enhanced depth imaging optical coherence tomography of choroidal nevus in 104 cases. Ophthalmology. 2012;119(5):1066-1072.

19. Espinoza G, Rosenblatt B, Harbour JW. Optical coherence tomography in the evaluation of retinal changes associated with suspicious choroidal melanocytic tumors. Am J Ophthalmol. 2004;137(1):90-95.

20. Materin MA, Raducu R, Bianciotto C, Shields CL. Fundus autofluorescence and optical coherence tomography findings in choroidal melanocytic lesions. Middle East Afr J Ophthalmol. 2010;17(3):201-206.

21. Shields CL, Furuta M, Mashayekhi A, et al. Clinical spectrum of choroidal nevi based on age at presentation in 3422 consecutive eyes. Ophthalmology. 2008;115(3):546-552.e2.

22. García-Arumí J, Amselem L, Gunduz K, et al. Photodynamic therapy for symptomatic subretinal fluid related to choroidal nevus. Retina. 2012;32(5):936-941.

23. Rundle P, Rennie I. Management of symptomatic choroidal naevi with photodynamic therapy. Eye (Lond). 2007;21(12):1531-1533.

24. Moon SJ, Wirostko WJ. Photodynamic therapy for extrafoveal choroidal neovascularization associated with choroidal nevus. Retina. 2006;26(4): $477-479$.

25. Cavalcante LL, Cavalcante ML, Murray TG, et al. Intravitreal injection analysis at the Bascom Palmer Eye Institute: evaluation of clinical indications for the treatment and incidence rates of endophthalmitis. Clin Ophthalmol. 2010;4:519-524.

\section{Dovepress}

PubMed Central and CAS, and is the official journal of The Society of Clinical Ophthalmology (SCO). The manuscript management system is completely online and includes a very quick and fair peer-review system, which is all easy to use. Visit http://www.dovepress.com/ testimonials.php to read real quotes from published authors. 